\title{
CLOSTRIDIUM SPORE ATTACHMENT TO HUMAN CELLS*
}

\author{
B. Panessa-Warren, and G. Tortora \\ Department of Clinical Laboratory Sciences, \\ School of Health Technology and Management, \\ SUNY, Stony Brook, NY 11794-8205 \\ and \\ J. Warren \\ Instrumentation Division \\ Brookhaven National Laboratory \\ Upton, NY 11973-5000
}

August, 1997

"Work supported in part by the U.S. Department of Energy Contract No. DE-AC02-98CH10886. 


\title{
CLOSTRIDIUM SPORE ATTACHMENT TO HUMAN CELLS
}

\author{
B. Panessa-Warren*, G.Tortora* and J. Warren**
}

* Department of Clinical Laboratory Sciences, School of Health Technology and Management, SUNY@ Stony Brook, New York 11794-8205

**Division of Instrumentation, Brookhaven National Laboratory, Upton, New York 11973

This paper uses high resolution scanning electron microscopy (SEM) with a LaB6 gun and the newest commercial field emission guns, to obtain high magnification images of intact clostridial spores throughout the activation/germination/outgrowth process. By high resolution SEM, the clostridial exosporial membrane can be seen to produce numerous delicate projections (following activation), that extend from the exosporial surface to a nutritive substrate (agar), or cell surface when anaerobically incubated in the presence of human cells (embryonic fibroblasts and colon carcinoma cells). Magnifications of 20,000 to $200,000 \mathrm{Xs}$ at accelerating voltages low enough to minimize or eliminate specimen damage (1-5 kV) have permitted the entire surface of C.sporogenes and C.difficile endospores to be examined during all stages of germination. The relationships between the spore and the agar or human cell surface were also clearly visible.

Clostridium sporogenes ATCC 3584 and C.difficile ATCC 43594 and 9689 were grown in cooked meat media, filtered and cleaned. Spore samples were fixed in $2.8 \%$ glutaraldehyde in $0.1 \mathrm{M}$ cacodylate buffer with $0.1 \% \mathrm{CaCl}_{2}$, post fixed in $1 \%$ aqueous $\mathrm{OsO}_{4}$, washed, dehydrated and critical point dried. Sucrose was added to glutaraldehyde fixative and cacodylate buffer solutions for preparations of human cells with spores. Cells grown on coverslips were placed in the incubator of the anaerobic hood and the media removed leaving a thin layer over the surface of the cells. Once the color indicator in the media became clear (anaerobic), 10 $\mu$ l of spore suspension was added to each coverslip. Spores were allowed to incubate for two, four, or six hours in the anaerobic hood. Samples were fixed in the hood, removed from the hood and left overnight in fresh fixative at $4^{\circ}-10^{\circ} \mathrm{C}$. Samples were washed in buffer, post fixed in $\mathrm{OsO}_{4}$, dehydrated and either infiltrated with plastic for thin sectioning, or dried by the critical point method. The coverslips were broken and small pieces attached to the specimen holder with silver paint.

For field emission microscopy (FEM), samples were coated with 0.1-3.3 $\mathrm{nm} \mathrm{W}$ or $\mathrm{Cr}$ using a rotary stage and crystal monitor. Specimens for LaB6 microscopy were coated with AuPd 5.0-10.0 $\mathrm{nm}$ in a sputter coater. C.sporogenes showed firm attachments to embryonic fibroblast cells. These attachments were not altered by washing, dehydration, drying or beam exposure and the spores often seemed connected by fibrillar material. The surface of the exosporium was covered with small delicate microvilli-like appendages. Even spores with emerging vegetative cells showed a complex surface architecture (Figure1,2). C.difficile spores did not attach to human fibroblasts, but showed a pronounced affinity for attaching to microvilli of the colon carcinoma cells. Field emission images were superior for high resolution detail of surface exosporial structures. The thin metal coating and low accelerating of FEM proved superior for spore imaging. However, serious charging problems arose when examining human cells attached to glass cover slips. Frame integration proved helpful in trying to obtain clear images of spores attached to cells. 


\section{AKNOWLEDGEMEN'T}

The authors would like to sincerely thank JEOL, Inc., and AMRAY Inc., for their assistance in doing the field emission work.

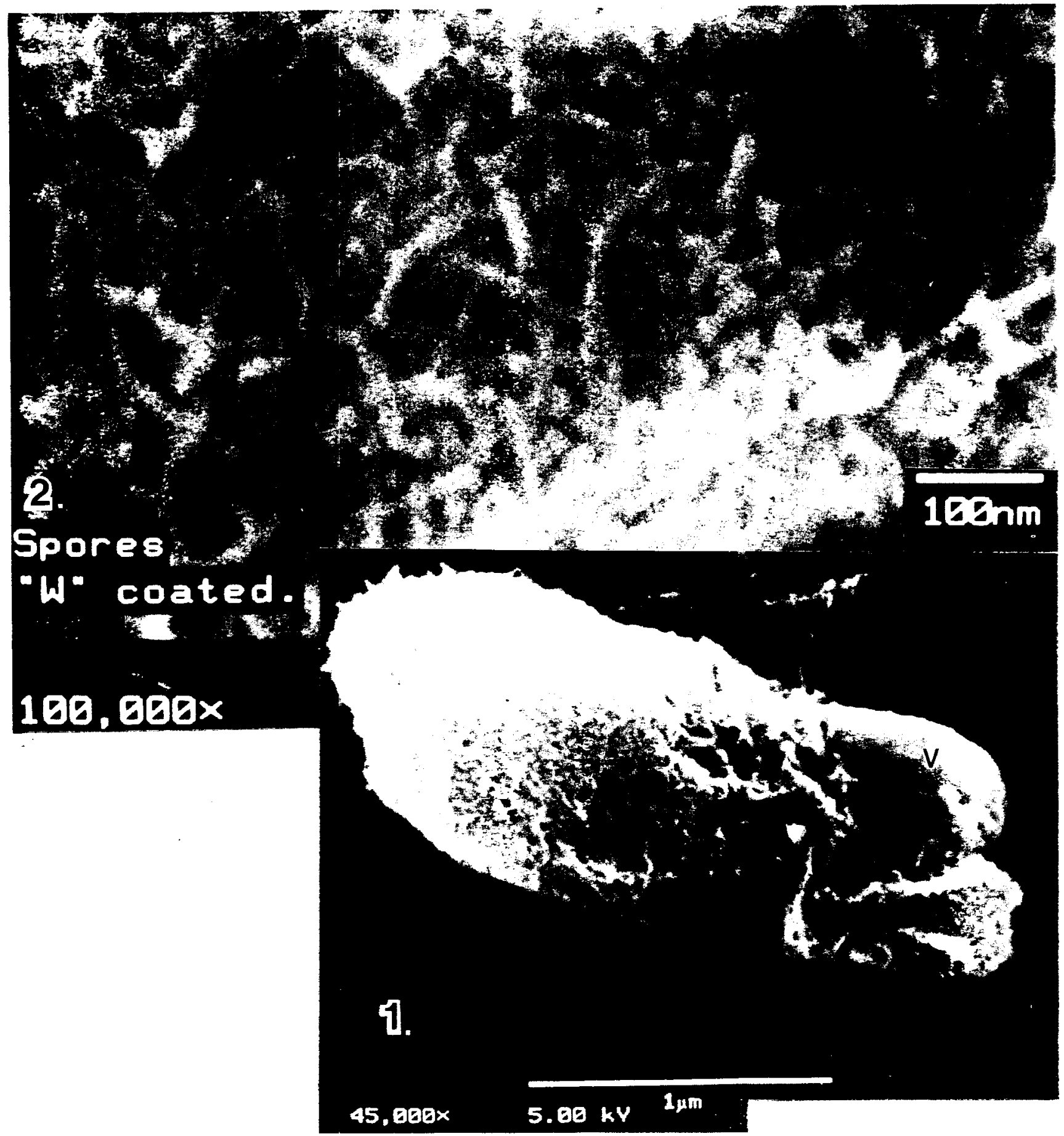

FIG 1. FEM images of C.sporogenes vegetative cell (V) emerging from exosporium covered with delicate "hair-like" projections during outgrowth.

FIG 2. At very high magnification $(100,000 \mathrm{X})$ the complex morphology of these profuse exosporial projections is clearly visible. Tungsten coated, $5 \mathrm{kV}$. 